\title{
Curcumin supplementation could improve diabetes-induced endothelial dysfunction associated with decreased vascular superoxide production and PKC inhibition
}

\author{
Sirada Rungseesantivanon ${ }^{1 \dagger}$, Naris Thenchaisri ${ }^{2 \dagger}$, Preecha Ruangvejvorachai ${ }^{3 \dagger}$, Suthiluk Patumraj ${ }^{4^{*}}$
}

\begin{abstract}
Background: Curcumin, an Asian spice and food-coloring agent, is known for its anti-oxidant properties. We propose that curcumin can improve diabetes-induced endothelial dysfunction through superoxide reduction.

Methods: Diabetes (DM) was induced in rats by streptozotocin (STZ). Daily curcumin oral feeding was started six weeks after the STZ injection. Twelve weeks after STZ injection, mesenteric arteriolar responses were recorded in real time using intravital fluorescence videomicroscopy. Superoxide and vascular protein kinase C (PKC- $\beta \|)$ were examined by hydroethidine and immunofluorescence, respectively.

Results: The dilatory response to acetylcholine (ACh) significantly decreased in DM arterioles as compared to control arterioles. There was no difference among groups when sodium nitroprusside (SNP) was used. ACh responses were significantly improved by both low and high doses (30 and $300 \mathrm{mg} / \mathrm{kg}$, respectively) of curcumin supplementation. An oxygen radical-sensitive fluorescent probe, hydroethidine, was used to detect intracellular superoxide anion $\left(\mathrm{O}_{2}{ }^{\bullet}\right)$ production. $\mathrm{O}_{2}{ }^{\bullet-}$ production was markedly increased in DM arterioles, but it was significantly reduced by supplementation of either low or high doses of curcumin. In addition, with a high dose of curcumin, diabetes-induced vascular PKC- $\beta \|$ expression was diminished.

Conclusion: Therefore, it is suggested that curcumin supplementation could improve diabetes-induced endothelial dysfunction significantly in relation to its potential to decrease superoxide production and PKC inhibition.
\end{abstract}

\section{Background}

Diabetes mellitus (DM) is characterized by chronic hyperglycemia and its developed diabetic complications, in particular, macroangiopathy and microangiopathy. These pathophysiological complications are often responsible for a decreased quality of life in diabetic patients [1]. Experimental evidence indicates that hyperglycemia induces a series of cellular events that increase the production of reactive oxygen species (ROS) [2]. In the vessel, one of the most important ROS is superoxide anion $(\mathrm{O} 2 \bullet-)$, which is formed by the univalent reduction of oxygen [3]. There are multiple enzymes involved

\footnotetext{
* Correspondence: suthilukp@yahoo.com

† Contributed equally

${ }^{4}$ Department of Physiology, Faculty of Medicine, Chulalongkorn University,

Bangkok 10330, Thailand

Full list of author information is available at the end of the article
}

in the production of $\mathrm{O} 2 \bullet$ - and its derivatives in the vasculature, in particular, vascular protein kinase $\mathrm{C}$ (PKC)activated $\mathrm{NAD}(\mathrm{P}) \mathrm{H}$ oxidase [4]. The $\mathrm{O} 2 \bullet$ - produced can inactivate nitric oxide $(\mathrm{NO})[5,6]$ directly, which leads to decreased NO bioavailability $[7,8]$. NO is an important molecule that involves many vascular functions. The diabetes-induced increase in $\mathrm{O} 2 \bullet-$ and its relation to diabetic vascular complications have attracted a lot of attention from several investigators. In animal models of diabetes, antioxidant defense capacities were diminished in certain tissues [9]. In addition, human and animal studies have attempted to restore vascular endothelial function using different types of antioxidants [10-12]. However, a critical evaluation of clinical trials suggests a difference in the ROS specific to various vascular diseases, thereby limiting the effectiveness of specific 
antioxidants [13]. Various herbal extracts are known to possess antioxidant properties. Curcumin, a yellow pigment from the root of Curcuma longa Linn., is a major component of turmeric and commonly used as a spice and food-coloring agent. Anti-oxidant and anti-inflammatory properties of curcumin have been well documented by previous studies [14-16]; however, the effect of curcumin, especially on diabetes-induced vascular $\mathrm{O}_{2}{ }^{\bullet-}$ production, remains to be clarified. Recently, it has been reported that curcumin $(300 \mathrm{mg} / \mathrm{kg})$ could enhance the effect of vitamin $C$ in protecting endothelial cells, through an anti-oxidant effect [17]. Therefore, in the present study, we tested the effects of curcumin supplementation by using two different doses of $30 \mathrm{mg} / \mathrm{kg}$ and $300 \mathrm{mg} / \mathrm{kg}$ on diabetes-induced endothelial dysfunction, which is associated with the direct effects on vascular $\mathrm{O}_{2}{ }^{\bullet-}$ production. We also examine the potential of curcumin in inhibiting diabetes-induced PKC-activation by using immunofluorescent staining.

\section{Methods}

Male Wistar rats were housed in a temperature- and light-controlled environment, fed standard chow and had acess to tap water ad libitum. The present study was conducted in accordance with the guidelines for animal experimentation established by the National Research Council of Thailand and approved by the Institutional Animal Care and Use Committee of Chulalongkorn University.

\section{Induction of diabetes}

The rats were randomly divided into non-diabetic and diabetic groups. Diabetes was induced by a single intravenous injection of streptozotocin $(55 \mathrm{mg} / \mathrm{kg}, \mathrm{STZ}$, Sigma-Aldrich Co., USA). STZ was freshly prepared by dissolving it in citrate buffer $(\mathrm{pH} 4.5$, Sigma-Aldrich Co., USA) and immediately injected into the tail vein after 8 hours of fasting. Control rats received citrate buffer of the same volume instead. STZ-induced diabetic rats were included and retained for the experiments if their blood glucose was greater than $200 \mathrm{mg} / \mathrm{dL}$. Blood glucose was measured by using a glucometer (ACCUCHEK, ADVANTAGE, Roche Diagnostics, Germany). Animals were separated in five groups: (1) diabetes (DM; n = 10), (2) DM-treated with curcumin (Cayman Chemical Co., USA) $30 \mathrm{mg} / \mathrm{kg}(\mathrm{DM}+\mathrm{cur} 30 ; \mathrm{n}=10)$, (3) DM-treated with curcumin $300 \mathrm{mg} / \mathrm{kg}$ (DM+cur300; $\mathrm{n}=10$ ), (4) control (con; $\mathrm{n}=10$ ), and (5) control treated with $300 \mathrm{mg} / \mathrm{kg}(\mathrm{con}+\operatorname{cur} 300 ; \mathrm{n}=10)$. It is noted that the daily oral feeding of curcumin was started at six weeks after the STZ injection, since it has been shown by our previous study that endothelial dysfunction in STZ-rats has already occurred at six-weeks after STZ injection [11].
Intravital observation of mesenteric arteriolar responses

Twelve weeks after STZ injection (or vehicle), the rats were anesthetized with an intraperitoneal injection of pentobarbital sodium $(50 \mathrm{mg} / \mathrm{kg}$ ). After tracheostomy, polyethylene tubes were inserted into the external jugular vein and the common carotid artery for injection of fluorescence tracers and monitoring of blood pressure, respectively. The abdominal cavity was opened via midline incision. The rat was placed on its right side on a microscope stage. A small loop of intestine was exteriorized. The mesentery was carefully spread on a plexiglass chamber with continuous perfusion by $1 \mathrm{~mL} / \mathrm{min}$ Krebs-Ringer buffer $\left(37^{\circ} \mathrm{C}\right.$, pH 7.4, bubbled with $95 \% \mathrm{O}_{2}$ and $5 \% \mathrm{CO}_{2}$, composition in mmol/L: $135.7 \mathrm{NaCl}, 4.7$ $\mathrm{KCl}, 2.52 \mathrm{CaCl}_{2}, 1.18 \mathrm{KH}_{2} \mathrm{PO}_{4}, 1.64 \mathrm{MgSO}_{4} .7 \mathrm{H}_{2} \mathrm{O}$, and 7.14 $\mathrm{NaHCO}_{3}$ ).

The second-order mesenteric arterioles (20 to $35 \mu \mathrm{m}$ in diameter) were viewed and recorded in real time by an epi-illumination fluorescence videomicroscopy system (Optiphot 2, Nikon, Japan) equipped with a $100 \mathrm{~W}$ mercury lamp, CCD camera (Hamamatsu C2400, Japan), a video recorder (VC-S5, Sharp, Japan) with a video timer (VTG-33, For-A, Japan) and a 20x objective lens (CF Plan Fluor, Nikon, Japan). Un-branched segments of mesenteric arterioles were visualized by fluorescein isothiocyanate-labeled dextran (FITC-Dextran 250, $5 \mu \mathrm{g} / \mathrm{mL}$, Sigma-Aldrich Co., USA). The mesenteric arterioles were allowed 15 minutes of stabilization and pre-constricted with norepinephrine $\left(10^{-5} \mathrm{~mol} / \mathrm{L}, \mathrm{NE}\right)$. Topical applications of two vasodilators, acetylcholine $\left(10^{-5} \mathrm{~mol} / \mathrm{L}, \mathrm{ACh}\right)$ and $\mathrm{NO}$ donor sodium nitroprusside $\left(10^{-5} \mathrm{~mol} / \mathrm{L}, \mathrm{SNP}\right)$ were used to test the functions of the endothelium and smooth muscle of mesenteric arterioles, respectively. Changes in mesenteric arteriolar diameters were analyzed off-line using digital image software (Image-Pro Plus; Media Cybernetics, Inc., USA) and expressed as the percentage of relaxation after preconstriction with NE by using the equation: $\left[\left(\mathrm{D}_{\mathrm{ACh}}{ }^{-}\right.\right.$ $\left.\left.\mathrm{D}_{\mathrm{NE}}\right) / \mathrm{D}_{\mathrm{NE}} \times 100\right]$.

\section{Direct detection of superoxide content using ethidium bromide fluorescence}

Mesenteric arterioles were subjected to $\mathrm{O}_{2}{ }^{\bullet-}$ measurement based on fluorescence detection using the DNAbinding fluorophore ethidium bromide (EB), which is formed by $\mathrm{O}_{2}{ }^{\bullet-}$ oxidation of hydroethidine (HE), using $490 \mathrm{~nm}$ excitation and $590 \mathrm{~nm}$ emission wavelengths [18]. The mesentery preparation was perfused with a buffer solution containing hydroethidine $\left(5.0 \times 10^{-6}\right.$ $\mathrm{mol} / \mathrm{L}$, Polysciences, USA) saturated with a $95 \% \mathrm{~N}_{2}$ and $5 \% \mathrm{CO}_{2}$ gas mixture for 60 minutes according to the modified method described by Suzuki et al. $[19,20]$. The number of nuclei labeled with ethidium bromide (EBpositive nuclei) along the mesenteric arteriolar wall was 


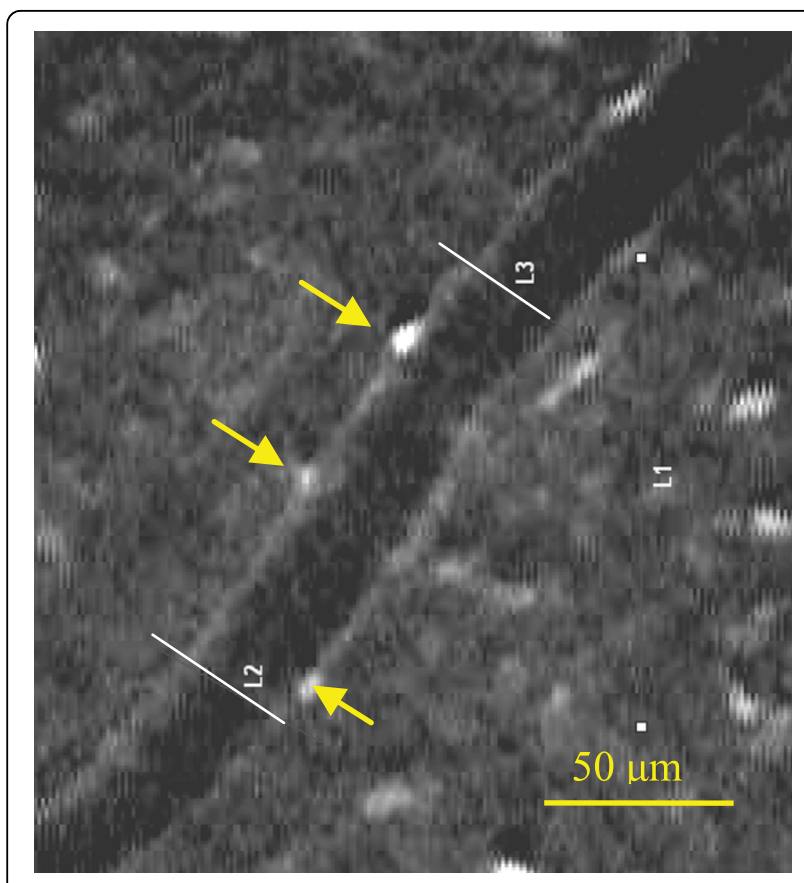

Figure 1 Ethidium bromide-positive nuclei. Number of ethidium bromide (EB)-positive nuclei from the selected arteriolar wall. The white lines depict the 100-micron vascular length where EB-positive nuclei were counted. (Bar represents $50 \mu \mathrm{m}$ ).

determined at 60 minutes after the onset of hydroethidine perfusion. The number of EB-stained nuclei was counted per 100 microns of vessel length, as shown in Figure 1 . The selection of microvessels was limited to arterioles with diameters ranging between 20 to $35 \mu \mathrm{m}$. The results were confirmed by the other examiner, who was blinded to grouping while counting.

\section{Immunofluorescent staining for PKC- $\beta$ II}

Single unbranched small mesenteric arteries with diameter $\sim 100 \mu \mathrm{m}$ were selected for study. The selected microvessels were post-fixed in $4 \%$ paraformaldehyde for 24 hours and were embedded in paraffin. These specimens were then deparaffinized in xylene, rehydrated in graded ethanol and distilled water, antigen unmasked with sodium citrate $(10 \mathrm{mmol} / \mathrm{L}, \mathrm{pH}$ 6.0, Dako, Denmark), and then exposed to a microwave heat source. Incubation with anti-PKC- $\beta$ II (1:100 dilution, Santa Cruz Biotechnology, CA) was performed at room temperature for 60 minutes. Sections were then washed in PBS and incubated with the secondary antibody swine anti-rabbit IgG-TRITC (1:50 dilution, Dako, Denmark) for 30 minutes at room temperature. Labeling of the arteries with secondary antibody alone was used as negative control. Images were obtained using laser scanning confocal microscopy (E800, Nikon, Japan) to establish the localization of PKC- $\beta$ II in small mesenteric arteries.

\section{Measurement of plasma glucose and HbA1c}

At the end of each experiment, a blood sample was collected from each rat for further plasma glucose and glycosylated hemoglobin (HbA1c) determination, using the enzymatic and turbidimetric immunoinhibition methods, respectively (Bangkok RIA Laboratory Co., Ltd., Bangkok, Thailand).

\section{Statistical analysis}

Data were expressed as means and standard errors of means (SEM). For comparison among groups, one-way analysis of variance (one-way ANOVA) and Tukey posthoc test were used. $P<0.01$ and $<0.05$ were considered statistically significant. All data were analyzed using the SPSS program (version 16.0) for Windows.

\section{Results}

Effects of curcumin on mean arterial blood pressure, plasma glucose, and $\mathrm{HbA} 1 \mathrm{c}$ levels

Twelve weeks after the injection of STZ, plasma glucose and $\mathrm{HbA} 1 \mathrm{c}$ values were significantly elevated in DM rats as compared with control rats (Table 1). Supplementation of curcumin for six weeks in control rats did not alter the plasma glucose level. Interestingly, the high-dose supplementation of curcumin in the DM +cur300 group significantly lowered the levels of both plasma glucose and HbA1c in comparison to the DM group $(P<0.05)$. The low dose of curcumin supplementation $(30 \mathrm{mg} / \mathrm{kg})$ resulted in a slight decrease in plasma glucose and significantly lower levels of HbA1c when compared to values observed in the DM group (DM = $10.73 \pm 0.32$, DM+cur30 $=8.20 \pm 0.88)(P<0.05)$. However, treatment with a high dose of curcumin $(300 \mathrm{mg} /$ $\mathrm{kg}$ ) resulted in only a $32 \%$ decrease in plasma glucose $(\mathrm{DM}=459.0 \pm 24.40, \mathrm{DM}+\mathrm{cur} 300=310.00 \pm 32.73)$, and the values of plasma glucose and $\mathrm{HbA} 1 \mathrm{c}$ of both

Table 1 Mean arterial blood pressure (mABP; $\mathbf{m m H g}$ ), plasma glucose $(\mathrm{mg} / \mathrm{dl})$, and glycosylated hemoglobin (HbA1c, \%)

\begin{tabular}{lccc}
\hline Group & $\begin{array}{c}\text { mABP } \\
(\mathbf{m m H g})\end{array}$ & $\begin{array}{c}\text { Plasma glucose }(\mathbf{m g} / \\
\mathbf{d L})\end{array}$ & HbA1c (\%) \\
\hline control & $103.1 \pm 3.57$ & $101.8 \pm 4.89$ & $3.68 \pm 0.17$ \\
con & $106.18 \pm 3.93^{\mathrm{NS}}$ & $106.8 \pm 0.92^{\mathrm{NS}}$ & $4.08 \pm 0.41^{\mathrm{NS}}$ \\
+ cur300 & & $459.0 \pm 24.40^{* *}$ & $10.73 \pm 0.32^{* *}$ \\
DM & $151.67 \pm 9.68^{* *}$ & $360.8 \pm 35.82^{* *}$ & $8.20 \pm 0.88^{* *}$, \\
DM+cur30 & $128.33 \pm 3.57$ & & + \\
& & $310.00 \pm 32.73^{* *}, \dagger$ & $7.90 \pm 0.97^{* *}$, \\
DM & $122.27 \pm 8.68+$ & & $\dagger$ \\
\hline cur300 & & & \\
\hline
\end{tabular}

Values are means \pm SEM $(n=10)$ from the groups of: 12-week STZ (DM), diabetes treated with curcumin (DM+cur30, DM+cur300), controls (con) and controls treated with curcumin (con+cur300). NS, no significant difference compared to control, ${ }^{* *} P<0.01$, significant difference compared to control and control treated with curcumin; $\dagger P<0.05$, significant difference from diabetic rats. 
$\mathrm{DM}+\mathrm{cur} 30$ and $\mathrm{DM}+\mathrm{cur} 300$ were significantly increased when compared to the controls $(P<0.01)$. Therefore, it is noted that the hyperglycemic state still existed in both groups, DM+cur30 and DM+cur300.

In comparison with the control group, mean arterial blood pressure (mABP) was significantly increased in the DM group $(P<0.01)$. This increased mABP was significantly attenuated with a high dose of curcumin supplementation $(P<0.05)$.

\section{Effects of curcumin on mesenteric arteriolar responses}

The dilatory response of the mesenteric arterioles to ACh $\left(10^{-5} \mathrm{~mol} / \mathrm{L}\right)$ was significantly decreased in the diabetic group $(8.11 \pm 0.44 \%)$ as compared to the control group $(12.82 \pm 0.2 \%, P<0.01)$ (figure 2$)$. Both low and high doses of curcumin supplementation (DM+cur30; $10.56 \pm 0.2 \%$ and DM+cur300; $11.88 \pm 0.52 \%)$ significantly restored arteriolar dilation in response to $\mathrm{ACh}$ $\left(10^{-5} \mathrm{~mol} / \mathrm{L}\right)$ in comparison to the physiology observed in DM rats $(8.11 \pm 0.44 \%, P<0.01)$. However, supplementation with curcumin in the con+cur300 group did not show any effects on ACh-induced arteriolar dilation as compared to control $(12.58 \pm 1.07 \%$ and $12.82 \pm$ $0.2 \%$, respectively).

Figure 3 demonstrated that the impaired vasodilatation in mesenteric arterioles of DM rats appeared to involve only endothelial cell function but not smooth muscle function because the vasodilation response to SNP-activation was not altered in DM (12.55 $\pm 0.95 \%)$, $\mathrm{DM}+\mathrm{cur} 30(14.71 \pm 0.38 \%)$, or $\mathrm{DM}+\mathrm{cur} 300$ groups $(13.54 \pm 1.40 \%)$.

\section{Effect of curcumin on vascular superoxide production}

By using hydroethidine-sensitive vascular superoxide, the results showed that the number of EB-positive nuclei per 100-micron vessel length were significantly increased along the vascular walls of DM rats (19.6 \pm $0.8)$ as compared to control rats $(4.4 \pm 0.6, P<0.01)$.
The numbers of EB-positive nuclei observed in DM +cur30 and DM+cur300 groups $(10.8 \pm 1.2$ and $11.2 \pm$ 1.8 , respectively) were significantly reduced as compared to DM $(19.6 \pm 1.8, P<0.01)$ but remained higher than levels observed in controls $(4.4 \pm 0.6, P<0.05)$ (figure 4$)$. There was no significant difference between the EBpositive nuclei in control and con+cur300 arterioles (4.4 \pm 0.6 and $5.2 \pm 0.4$ respectively).

\section{Effect of curcumin on PKC- $\beta$ II in mesenteric artery}

Immunofluorescent staining of small mesenteric arteries displayed a strong PKC- $\beta$ II signal in DM rats (figure $5 C)$. In contrast, the TRITC signals of anti-PKC- $\beta$ II antibodies were weak in control and $\mathrm{DM}+$ cur rat vessels (figure 5B and 5D, respectively). Negative controls displayed minimal detectable fluorescence when the secondary antibodies were used alone (figure 5A).

\section{Correlation between intracellular superoxide production and arteriolar vasodilation}

To examine the correlation between intracellular superoxide production and endothelial vascular response, figure 6 shows the relationship between superoxide production and ACh-induced arteriolar vasodilation, for every group. The results indicated that the EB-positive nuclei along the mesenteric arterioles and the percent changes in arteriolar diameters stimulated by ACh have a significant correlation $(0.78, P<0.01)$. This correlation is described by the linear equation:

$$
y=0.29 x+14.5\left(R^{2}=0.78, P<0.01\right)
$$

Where $\mathrm{x}$ is the number of EB-positive nuclei per 100 um vessel length and $y$ is the percentage change in arteriolar diameter.

\section{Discussion}

In the present study, we have shown that the effect of curcumin supplementation on diabetes-induced

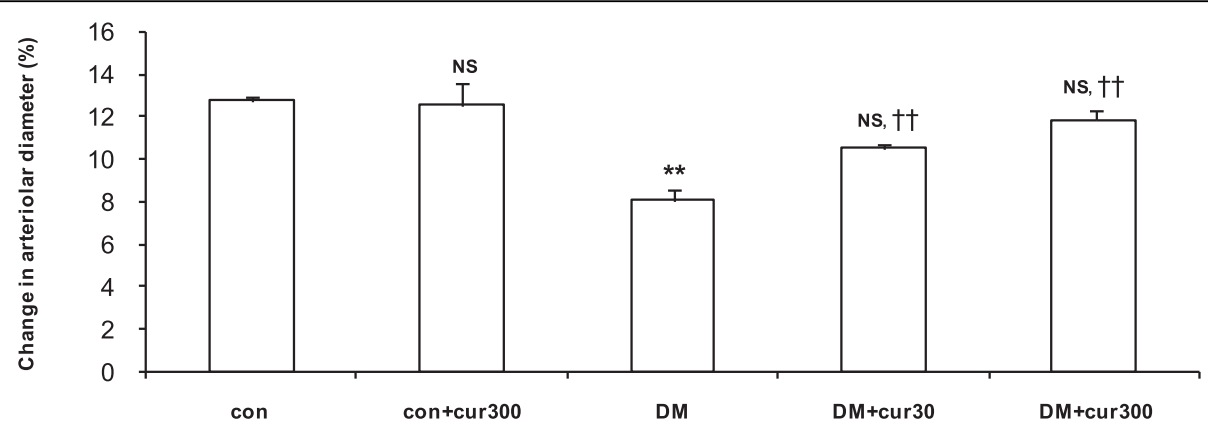

Figure 2 Acetylcholine-induced arteriolar vasodilation. Acetylcholine-induced changes in mesenteric arteriolar diameters from control (con), diabetes (DM) and curcumin-treated groups (DM+cur30, DM+cur300 and con+cur300). Data are means \pm SEM ( $\mathrm{n}=5$ for each group). NS, not significant different compared to control arterioles; ${ }^{* *} P<0.01$, significant difference compared to control arterioles; $†+P<0.01$, significant difference compared to diabetic arterioles. 


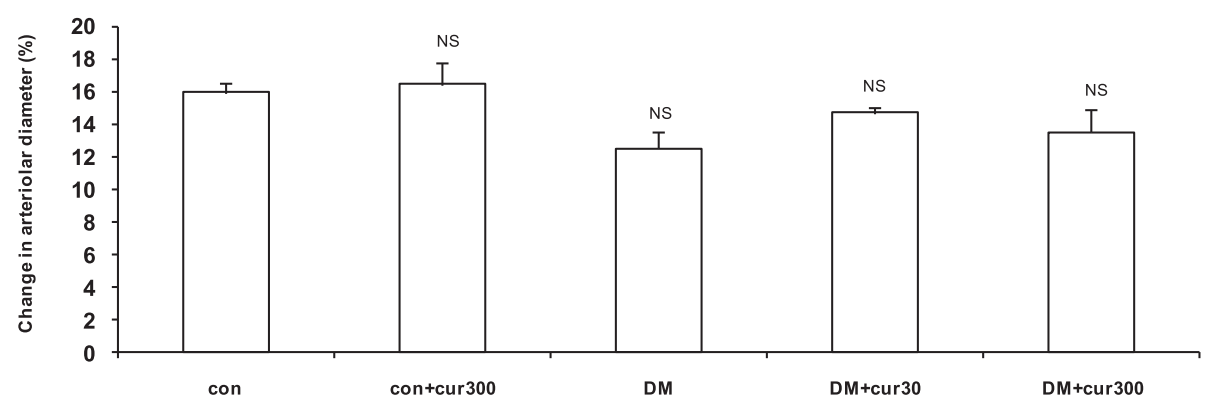

Figure 3 Sodium nitroprusside-induced arteriolar vasodilation. Sodium nitroprusside-induced changes in mesenteric arteriolar diameter from control (con), diabetes (DM) and curcumin-treated groups (con+cur300, DM+cur30, DM+cur300). Data are means \pm SEM ( $n=5$ for each group). NS, no significant difference compared to control arterioles.

endothelial dysfunction is closely associated with its potential as an anti-oxidant. The supplementation of either low or high doses of curcumin appears to improve diabetic endothelial dysfunction, as shown by the increase in ACh-activated vasodilation. However, there was no significant difference between low and high doses in terms of restoring effects. In contrast to this increase in ACh-vasorelaxation, endothelium-independent relaxation in response to the NO donor SNP was not affected by either diabetes or curcumin supplementation. Therefore in this twelve-week model of diabetes, the NO-stimulated cGMP signaling in arteriolar smooth muscle was not the primary target of treatment.

It is well established that hyperglycemia can produce ROS production by a series of cellular events and further leads to diabetic complications due to oxidative stress [2,21]. Previously, a diabetes-induced increase in ROS was indirectly demonstrated using lipid peroxidation end-products (e.g., malondialdehyde [MDA]) as an indicator $[10,17]$.

In order to examine the dynamic process of diabetesstimulated ROS production and its correlation with endothelial dysfunction, our study utilized hydroethidine-sensitive vascular superoxide detection. One of the most frequently used assays for the detection of cellular $\mathrm{O}_{2}{ }^{\bullet-}$ production utilizes hydroethidine as an intracellular probe $[22,23]$. In the presence of $\mathrm{O}_{2}{ }^{\bullet-}$, hydroethidine is rapidly converted to ethidium bromide, which binds to DNA and is detected by its red fluorescent light following minimal oxidation induced by $\mathrm{H}_{2} \mathrm{O}_{2}, \mathrm{ONOO}^{-}$, or $\mathrm{HOCl}^{-}[24]$. In situ nuclei labeled with ethidium bromide along the arteriolar wall could be observed and the

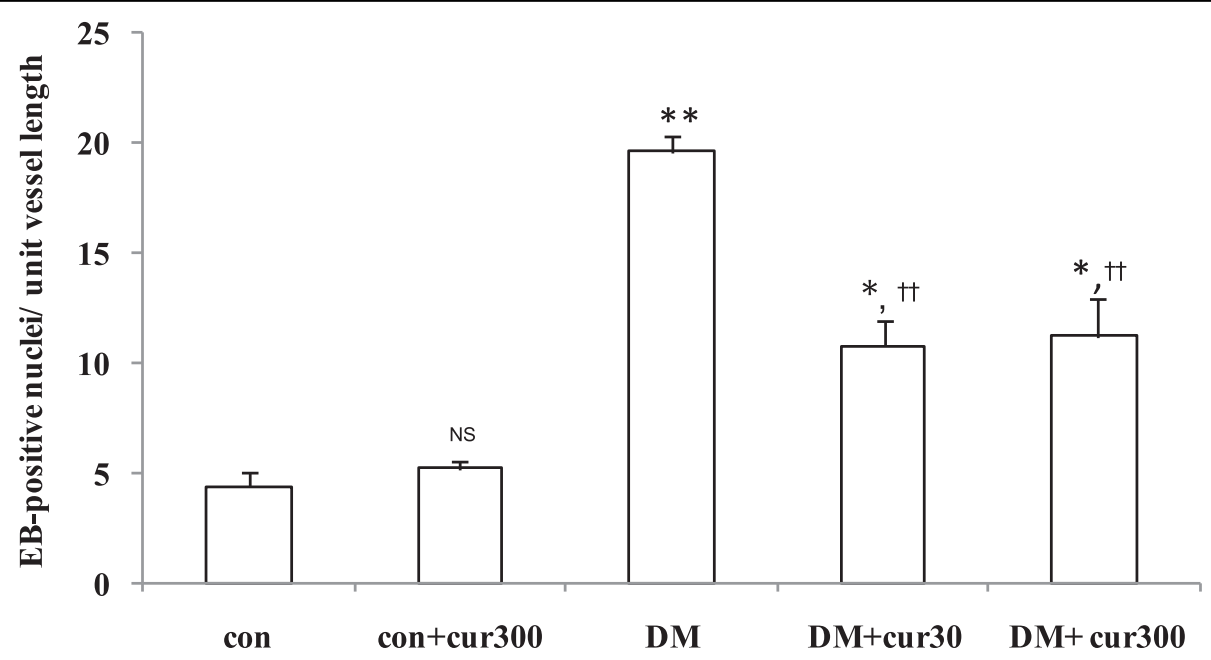

Figure 4 Number of ethidium bromide-positive nuclei. Histogram showing the ethidium bromide-positive nuclei along the mesenteric arterioles of rats that were untreated diabetics (DM), diabetics treated with low curcumin (DM+cur30), diabetics treated with high curcumin (DM + cur300), controls (con) or controls treated with curcumin (con+cur300) rats. Data are expressed as mean \pm SEM ( $n=5$ for each group). NS, no significant difference compared to control arterioles; ${ }^{* *} P<0.01$ and ${ }^{*} P<0.05$, significant difference compared to control arterioles; $†+P<0.01$, significant difference compared to diabetic arterioles. 

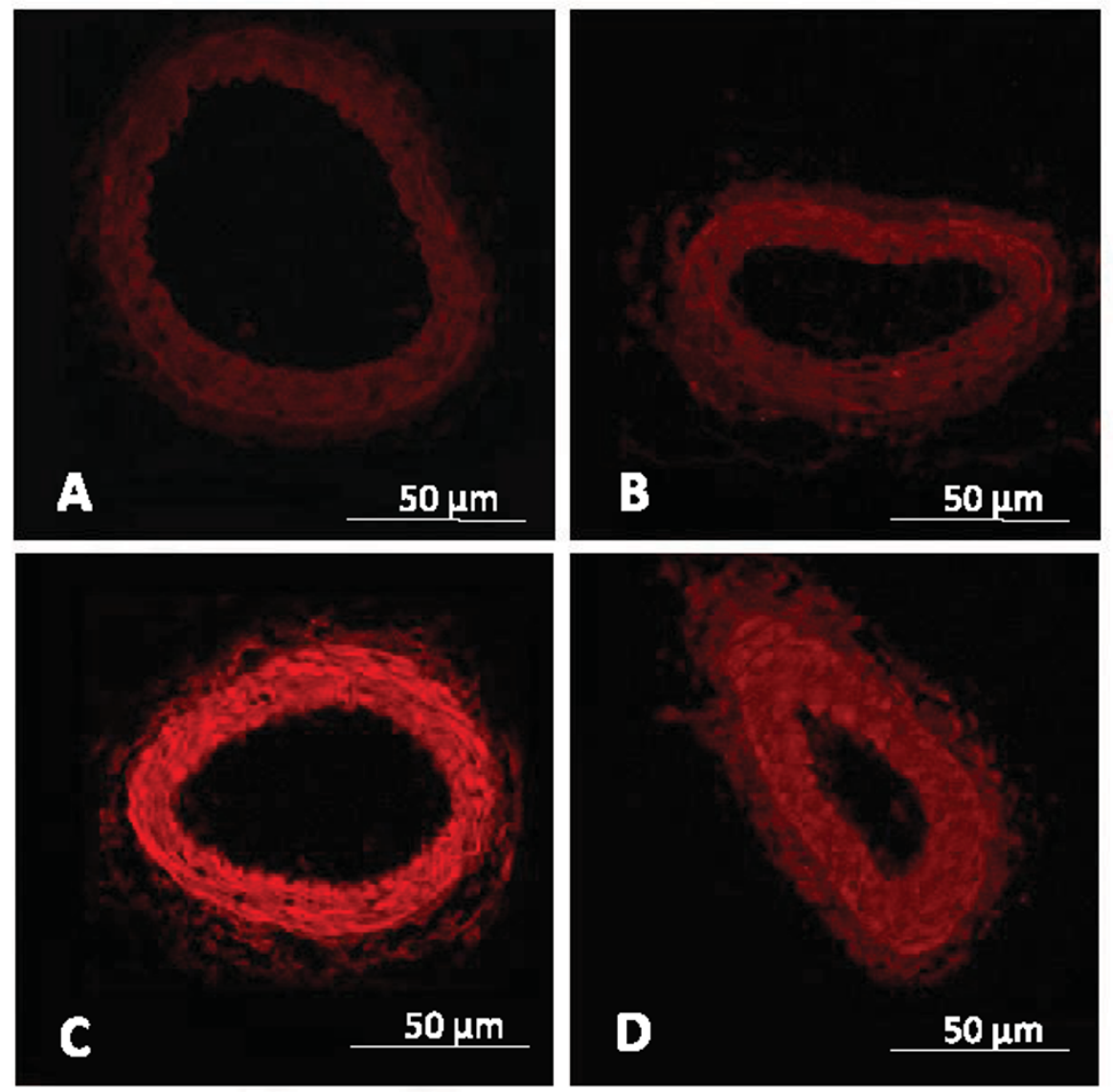

Figure 5 Immunofluorescent staining for PKC- $\beta$ II. PKC- $\beta \|$ and TRITC signals from immunofluorescent staining of mesenteric arteries. Microvessels with diameter of approximately $100 \mu \mathrm{m}$ were fixed in $4 \%$ paraformaldehyde for 24 hours and then embedded in paraffin. They were later deparaffinized in xylene and rehydrated in a mixture of ethanol and distilled water. Antigens were unmasked using sodium citrate (10 $\mathrm{mmol} / \mathrm{L}, \mathrm{pH}$ 6.0), followed by exposure to a microwave heat source. Samples were then incubated at room temperature for 60 minutes with anti-PKC- $\beta \|$ at 1:100. Sections were washed in PBS and incubated with swine anti-rabbit lgG-TRITC (1:50 in PBS) for 30 minutes at room

temperature. Immunofluorescent staining of small mesenteric arteries displayed a strong signal for PKC- $\beta \|$ in DM rats (Figure 5C). In contrast, the TRITC signals of anti-PKC- $\beta \|$ antibodies were weak in the controls and DM+cur rat vessels (Figure 5 B and D, respectively). The negative control displayed a minimal detectable fluorescence when the secondary antibodies were used alone (Figure 5A).

number of EB-sensitive nuclei could be quantitatively estimated per 100 microns of vessel length, as shown in Figure 4.

The results showed that superoxide production along the arteriolar wall was about 4.45 times higher in DM rats than controls. This excessive increase in vascular superoxide may have destroyed the vascular endothelial lining, yielding a 0.67-fold decrease in Ach response.

Interestingly, our findings have indicated that this 4.45 -fold increase in superoxide production in diabetic vasculature could be attenuated by daily oral curcumin supplementation. However, there was no difference between the low and high doses of curcumin in terms of reducing superoxide production at the diabetic vascular wall. Both doses of curcumin examined in this study were able to decrease superoxide production by almost two-fold. Curcumin has been reported as a potent scavenger of a variety of ROS [25], exhibiting antiinflammatory activity as well as antioxidant properties [17,26-29]. The phenolic $(\mathrm{OH})$ structure of curcumin was believed to be essential for curcumin's anti-oxidant activity [28].

In addition, the anti-oxidant effect of curcumin on protecting endothelial function against ROS damage may be partially attributed to the hypoglycemic effect of curcumin. Our findings are consistent with others showing that treatment with curcumin in diabetic rats leads to lower plasma glucose levels $[17,29]$. However, the new finding in the present study was that the hypoglycemic potential of curcumin is dose dependent (Table 1). 


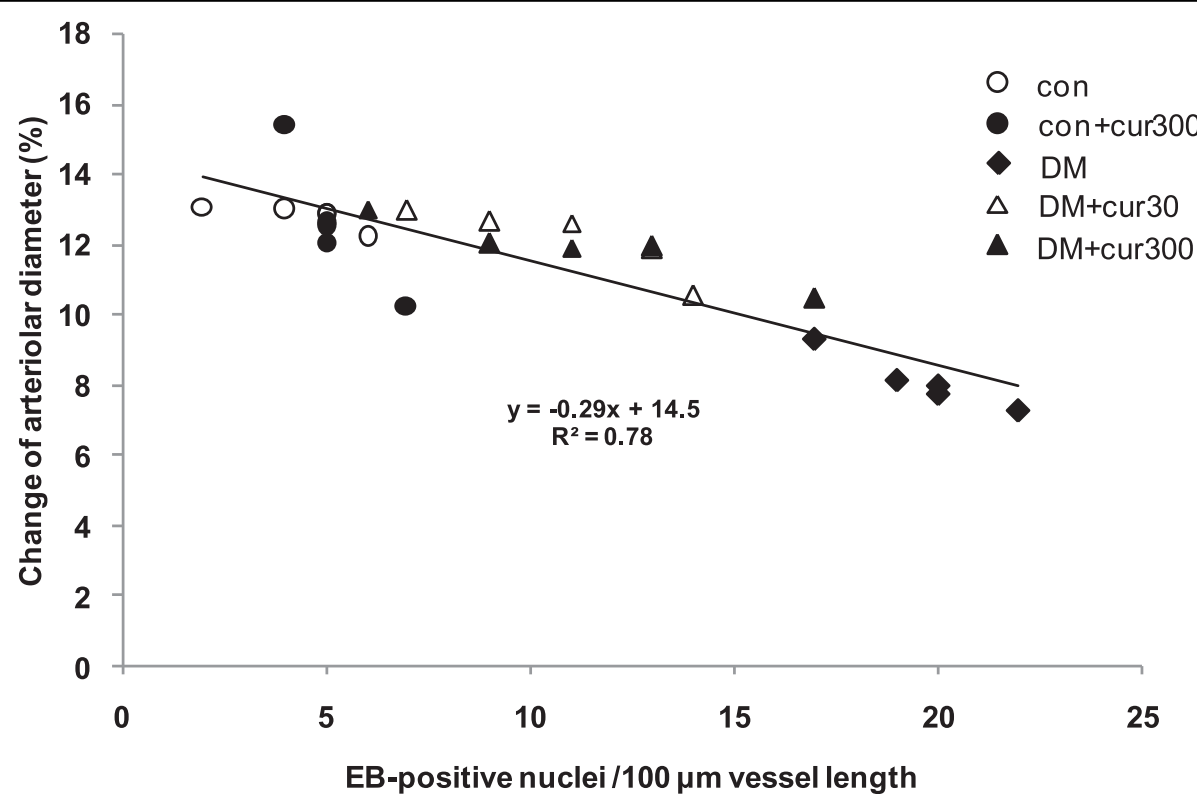

Figure 6 Relationship between ethidium bromide-positive nuclei and \% changes in ACh-induced arteriolar vasodilation. Relationship between EB-positive nuclei per $100 \mu \mathrm{m}$ vessel length and percentage of ACh-induced change in arteriolar diameter for diabetes (DM), diabetes treated with 30 and $300 \mathrm{mg} / \mathrm{kg}$ curcumin (DM+cur30 and DM+cur300, respectively), control (con) and control treated with $300 \mathrm{mg} / \mathrm{kg}$ curcumin (con+cur300).

Since HbA1c is the product of non-enzymatic glycosylation, therefore, it is mostly a better indicator of glycemic control than plasma glucose level. Although the mechanisms underlying the anti-diabetic action of curcumin remain unknown, it has been suggested by other investigators that curcumin may inhibit hepatic glucose output and/or stimulate insulin secretion from the pancreas [26,30,31]. Moreover, it has been reported recently that the anti-diabetic potential of anti-oxidants such as vitamin $\mathrm{C}$ could protect glucose transporter 1 (GLUT-1) [32]. Therefore, the hypoglycemic effect of curcumin may be attributed to this effect on GLUT-1 as well.

STZ induces cytotoxicity mediated by reactive oxygen species, as evidenced by B cell damage. Therefore, it is possible that the hypoglycemic effect of curcumin, mediated by stimulating insulin secretion from the pancreas, may be limited by the number of $B$ cells remaining. Therefore, the antidiabetic effect of curcumin should be further clarified with the understanding that curcumin supplementation cannot be used as an antidiabetic on its own.

The increase in hyperglycemia-induced oxygen-derived free radicals was believed to be a major contributor to the reduction of $\mathrm{NO}$ bioavailability observed in diabetes. The interaction between $\mathrm{NO}$ and $\mathrm{O}_{2}{ }^{\bullet-}$ occurs at an extremely rapid rate, three times faster than the rate of $\mathrm{O}_{2}{ }^{\bullet-}$ reaction with SOD [33]. Therefore, this hyperglycemia-induced $\mathrm{O}_{2}^{\bullet-}$ may quench $\mathrm{NO}$, preventing endothelium-dependent vasodilation [34-36].
Our findings showed that both low and high doses of curcumin could significantly lower blood glucose by $18.73 \%$ and $30.26 \%$, respectively, in the diabetic group. In addition, the results also showed that both low and high doses of curcumin could decrease diabetic vascular superoxide production down by $55.1 \%$ and $57.1 \%$, respectively. Simultaneously, both doses of curcumin were able to increase ACh-activated vasodilatation by up to $30.22 \%$ and $46.47 \%$, respectively. Although our study did not monitor NO production directly, this parameter can be measured indirectly by quantifying endothelialdependent vasodilation. Our findings in this regard indicate that curcumin supplementation could enhance endothelial-dependent relaxation in diabetic rats. Johnson et al. used in vitro studies to determine that the mechanism of curcumin-mediated protection against NO oxidation involves the sequestration of reaction intermediates [35]. The $\mathrm{IC}_{50}$ for curcumin with $1.0 \mu \mathrm{M}$ DEA/NO was calculated to be $13 \mu \mathrm{M}$. Moreover, the authors also suggested that the mechanism of curcumin action involved the sequestration of $\mathrm{NO}_{2}$ but not $\mathrm{NO}$.

Previous studies in rat aortic rings also demonstrated that curcumin $(10(-11) \mathrm{mol} / \mathrm{L})$ could alleviate the acute increase in glucose levels induced by dysfunctional endothelium-dependent vasodilation [37]. The authors suggested that the effect of curcumin may be due to its ability to enhance heme oxygenase and guanylate cyclase (GC) activity. Ach as well as NO-stimulated cGMP signaling is required for normal endothelium-dependent 
vasodilation. Therefore, the protective effect of curcumin on vasodilatation could be completely eliminated by the non-selective guanylate cyclase (GC) inhibitor methylene blue [37].

A clinical study by Usharani et al. (2008) [38] showed that NCB-02 (two capsules containing curcumin $150 \mathrm{mg}$ twice daily) significantly reduced the levels of malondialdehyde, ET-1, IL- 6 and TNF[alpha] in type 2 diabetes patients. Therefore, the molecular mechanisms of curcumin-mediated increases in vascular NO bioavailability might be enhanced by its anti-oxidant properties and by its anti-inflammatory effects. Notably, the pharmacokinetics of curcumin have recently been found to be associated with many pathophysiologies via its actions on signaling networks such as the NF- $\kappa \mathrm{B}$ and MAPK/ERK pathways $[39,40]$.

Numerous studies have shown that both free fatty acids and high glucose levels in diabetes may activate PKC in various vascular cells via de novo synthesis of diacylglycerol (DAG) [41]. In addition, it has been reported that such activated PKC could facilitate increased $\mathrm{O}_{2}{ }^{\bullet-}$ production through $\mathrm{PKC}$-dependent activation of $\mathrm{NAD}(\mathrm{P}) \mathrm{H}$ oxidase in vascular cells $[42,43]$. Activated PKC result in sustained increases in the production of $\mathrm{O}_{2}{ }^{\bullet-}$ and induce oxidative damage to diabetic blood vessels, and it also induces a number of pathogenic consequences by activating NF- $\kappa \mathrm{B}$ and affecting the expression of endothelial nitric oxide synthetase (eNOS), endothelin-1(ET-1), vascular endothelial growth factor (VEGF), transforming growth factor- $\beta$ (TGF- $\beta$ ) and plasminogen activator inhibitor-1 (PAI-1) $[42,44]$. Therefore, in the present study, the effect of highdose curcumin on activated PKC was further investigated. Immunofluorescent micrographs revealed that diabetesactivated PKC expression was increased markedly in 12week diabetic mesenteric arterial wall. Interestingly, the immunofluorescent micrograph indicated that curcumin supplementation, at a dose of $300 \mathrm{mg} / \mathrm{kg}$, could suppress this diabetes-activated PKC expression (Figure 5D). This finding is in agreement with the previous report by Balasubramanyam et al. (2003), which determined that the dosedependent ROS inhibitory effect of curcumin interfered mechanistically with PKC activity [45].

In order to confirm the importance of curcumin action, the correlation between HE-sensitive superoxide production and $\mathrm{ACh}$-induced arteriolar vasodilation was examined for all five groups. These results were confirmed by the strong correlation between both parameters $(0.78, P<0.01)$.

\section{Conclusion}

In conclusion, diabetes-induced endothelial dysfunction is closely associated with increases in oxidative stress along the vascular wall. Curcumin supplementation can improve diabetes-induced endothelial dysfunction through its ability to decrease $\mathrm{O}_{2} \bullet$ production by inhibiting PKC. Curcumin supplementation may benefit diabetic patients by improving microvascular function and preventing cardiovascular complications.

\section{Acknowledgements}

This study was supported by the $90^{\text {th }}$ Anniversary of Chulalongkorn University Fund (Ratchadaphiseksomphot Endowment Fund).

\section{Author details}

${ }^{1}$ Inter-department of Physiology, Graduate School, Chulalongkorn University, Bangkok 10330, Thailand. 'Department of Companion Animal Clinical Sciences, Faculty of Veterinary Medicine, Kasetsart University, Bangkok 10900, Thailand. ${ }^{3}$ Department of Pathology, Faculty of Medicine, Chulalongkorn University, Bangkok 10330, Thailand. ${ }^{4}$ Department of Physiology, Faculty of Medicine, Chulalongkorn University, Bangkok 10330, Thailand.

\section{Authors' contributions}

SR: Carried out the animal experiments, including Intra-vital set-up and immunoassays, and edited the first draft of the manuscript. ${ }^{1 *}$ Contributed $45 \%$ of this work. NT: Participated in the experimental design and manuscript improvement. ${ }^{2^{*}}$ Contributed $15 \%$ of this work. PR: Carried out sample selection and fixation. ${ }^{3 *}$ Contributed $5 \%$ of this work. SP: Grant support and manuscript improvement, submission, and correspondence. ${ }^{45}$ Contributed $35 \%$ of this work. All authors read and approved the final version of the manuscript.

\section{Competing interests}

The authors declare that they have no competing interests.

Received: 12 October 2009 Accepted: 14 October 2010 Published: 14 October 2010

\section{References}

1. UPDSU Group: Intensive blood-glucose control with sulphonylureas or insulin compared with conventional treatment and risk of complications in patients with type 2 diabetes (UKPDS 33). Lancet 1998, 352(9131):837-53.

2. Guzik TJ, Mussa S, Gastaldi D, Sadowski J, Ratnatunga C, Pillai R, et al: Mechanisms of increased vascular superoxide production in human diabetes mellitus: role of $\mathrm{NAD}(\mathrm{P}) \mathrm{H}$ oxidase and endothelial nitric oxide synthase. Circulation 2002, 105(14):1656-62.

3. Droge W: Free radicals in the physiological control of cell function. Physiol Rev 2002, 82(1):47-95.

4. Hink U, Li H, Mollnau H, Oelze M, Matheis E, Hartmann M, et al: Mechanisms underlying endothelial dysfunction in diabetes mellitus. Circ Res 2001, 88(2):E14-22.

5. Katusic ZS: Superoxide anion and endothelial regulation of arterial tone. Free Radic Biol Med 1996, 20(3):443-8.

6. Rubanyi GM, Vanhoutte PM: Superoxide anions and hyperoxia inactivate endothelium-derived relaxing factor. Am J Physiol 1986, 250(5 Pt 2): H822-7.

7. Lund DD, Faraci FM, Miller FJ Jr, Heistad DD: Gene transfer of endothelial nitric oxide synthase improves relaxation of carotid arteries from diabetic rabbits. Circulation 2000, 101(9):1027-33.

8. Mugge A, Elwell JH, Peterson TE, Hofmeyer TG, Heistad DD, Harrison DG: Chronic treatment with polyethylene-glycolated superoxide dismutase partially restores endothelium-dependent vascular relaxations in cholesterol-fed rabbits. Circ Res 1991, 69(5):1293-300.

9. Wohaieb SA, Godin DV: Alterations in free radical tissue-defense mechanisms in streptozocin-induced diabetes in rat. Effects of insulin treatment. Diabetes 1987, 36(9):1014-8.

10. Jariyapongskul A, Patumraj S, Yamaguchi S, Niimi H: The effect of longterm supplementation of vitamin $C$ on leukocyte adhesion to the cerebral endothelium in STZ-induced diabetic rats. Clin Hemorheol Microcirc 2002, 27(1):67-76.

11. Sridulyakul P, Chakraphan D, Patumraj S: Vitamin C supplementation could reverse diabetes-induced endothelial cell dysfunction in mesenteric microcirculation in STZ-rats. Clin Hemorheol Microcirc 2006, 34(1-2):315-21. 
12. Timimi FK, Ting HH, Haley EA, Roddy MA, Ganz P, Creager MA: Vitamin C improves endothelium-dependent vasodilation in patients with insulindependent diabetes mellitus. J Am Coll Cardiol 1998, 31(3):552-7.

13. Taniyama $Y$, Griendling KK: Reactive oxygen species in the vasculature: molecular and cellular mechanisms. Hypertension 2003, 42(6):1075-81.

14. Bengmark S: Curcumin, an atoxic antioxidant and natural NFkappaB, cyclooxygenase-2, lipooxygenase, and inducible nitric oxide synthase inhibitor: a shield against acute and chronic diseases. JPEN J Parenter Enteral Nutr 2006, 30(1):45-51

15. Masuda T, Maekawa T, Hidaka K, Bando H, Takeda Y, Yamaguchi H: Chemical studies on antioxidant mechanism of curcumin: analysis of oxidative coupling products from curcumin and linoleate. J Agric Food Chem 2001, 49(5):2539-47.

16. Masuda T, Toi Y, Bando H, Maekawa T, Takeda Y, Yamaguchi H: Structural identification of new curcumin dimers and their contribution to the antioxidant mechanism of curcumin. J Agric Food Chem 2002, 50(9):2524-30

17. Patumraj $S$, Wongeakin $N$, Sridulyakul $P$, Jariyapongskul $A$, Futrakul $N$, Bunnag S: Combined effects of curcumin and vitamin $\mathrm{C}$ to protect endothelial dysfunction in the iris tissue of STZ-induced diabetic rats. Clin Hemorheol Microcirc 2006, 35(4):481-9.

18. Thomas $G$, Roques B: Proton magnetic resonance studies of ethidium bromide and its sodium borohydride reduce derivative. FEBS Letters 1972, 26:169-75.

19. Suzuki H, DeLano FA, Parks DA, Jamshidi N, Granger DN, Ishii $H$, et al: Xanthine oxidase activity associated with arterial blood pressure in spontaneously hypertensive rats. Proc Natl Acad Sci USA 1998, 95(8):4754-9.

20. Suzuki H, Swei A, Zweifach BW, Schmid-Schonbein GW: In vivo evidence for microvascular oxidative stress in spontaneously hypertensive rats. Hydroethidine microfluorography. Hypertension 1995, 25(5):1083-9.

21. Brownlee M: Biochemistry and molecular cell biology of diabetic complications. Nature 2001, 414(6865):813-20

22. Bizyukin A, Soodaeva S: Study of oxidative phagocyte metabolism using the fluorescent indicator hydroethidine. Pharmaceu Chem J 1995, 29:236-40.

23. Carter WO, Narayanan PK, Robinson JP: Intracellular hydrogen peroxide and superoxide anion detection in endothelial cells. J Leukoc Biol 1994, 55(2):253-8

24. Benov L, Sztejnberg L, Fridovich I: Critical evaluation of the use of hydroethidine as a measure of superoxide anion radical. Free Radic Biol Med 1998, 25(7):826-31.

25. Reddy AC, Lokesh BR: Studies on the inhibitory effects of curcumin and eugenol on the formation of reactive oxygen species and the oxidation of ferrous iron. Mol Cell Biochem 1994, 137(1):1-8.

26. Halim E, Hussain M: Hypoglycemic, hypolipidemic and antioxidant properties of combination of Curcumin from CURCUMA LONGA, Linn, and partially purified product from ABROMA AUGUSTA, Linn, in streptozotocin induced diabetes. Indian J Clin Biochem 2002, 17:33-43.

27. Kunchandy E, Rao M: Oxygen radical scavenging activity of curcumin. Int J Pharm 1990, 58(3):237-40

28. Priyadarsini KI, Maity DK, Naik GH, Kumar MS, Unnikrishnan MK, Satav JG, et al: Role of phenolic $\mathrm{O}-\mathrm{H}$ and methylene hydrogen on the free radical reactions and antioxidant activity of curcumin. Free Radic Biol Med 2003, 35(5):475-84.

29. Sharma S, Kulkarni SK, Chopra K: Curcumin, the active principle of turmeric (Curcuma longa), ameliorates diabetic nephropathy in rats. Clin Exp Pharmacol Physiol 2006, 33(10):940-5.

30. Lochhead PA, Salt IP, Walker KS, Hardie DG, Sutherland C: 5aminoimidazole-4-carboxamide riboside mimics the effects of insulin on the expression of the 2 key gluconeogenic genes PEPCK and glucose-6phosphatase. Diabetes 2000, 49(6):896-903.

31. Yamauchi T, Kamon J, Minokoshi Y, Ito Y, Waki H, Uchida S, et al: Adiponectin stimulates glucose utilization and fatty-acid oxidation by activating AMP-activated protein kinase. Nat Med 2002, 8(11):1288-95.

32. Kim DI, Lim SK, Park MJ, Han HJ, Kim GY, Park SH: The involvement of phosphatidylinositol 3-kinase/Akt signaling in high glucose-induced downregulation of GLUT-1 expression in ARPE cells. Life Sci 2007, 80(7):626-32.
33. Benz D, Cadet P, Mantione K, Zhu W, Stefano G: Tonal nitric oxide and health-a free radical and a scavenger of free radicals. Med Sci Monit 2002, 8(1):RA1-4.

34. Jariyapongskul A, Patumraj $S$, Niimi H: Cerebral endothelial dysfunction in diabetes: intravital microscopic analysis using streptozotocin-induced diabetic rats. Clin Hemorheol Microcirc 2003, 29(3-4):331-5.

35. Johnston BD, DeMaster EG: Suppression of nitric oxide oxidation to nitrite by curcumin is due to the sequestration of the reaction intermediate nitrogen dioxide, not nitric oxide. Nitric Oxide 2003, 8(4):231-4.

36. Meininger CJ, Marinos RS, Hatakeyama K, Martinez-Zaguilan R, Rojas JD, Kelly KA, et al: Impaired nitric oxide production in coronary endothelial cells of the spontaneously diabetic $\mathrm{BB}$ rat is due to tetrahydrobiopterin deficiency. Biochem J 2000, 349(Pt 1):353-6.

37. Fang XD, Yang F, Zhu L, Shen YL, Wang LL, Chen YY: Curcumin ameliorates high glucose-induced acute vascular endothelial dysfunction in rat thoracic aorta. Clin Exp Pharmacol Physiol 2009, 36(12):1177-82.

38. Usharani P, Mateen A, Naidu M, Raju Y, Chandra N: Effect of NCB-02, Atorvastatin and placebo on endothelial function, oxidative stress and inflammatory markers in patients with $\mathrm{T} 2$ diabetes mellitus: A randomized, parallel-group, placebo-controlled, 8-week study. Drug in R\&D 2008, 9(4):243-50.

39. Aggarwal BB, Sung B: Pharmacological basis for the role of curcumin in chronic diseases: an age-old spice with modern targets. Trends Pharmacol Sci 2009, 30(2):85-94.

40. Patumraj S, Yoysungneon P: Curcumin as a therapeutic agent against cancer. Asian Biomedicine 2007, 1:239-52.

41. Beckman JA, Goldfine AB, Gordon MB, Garrett LA, Creager MA: Inhibition of protein kinase Cbeta prevents impaired endothelium-dependent vasodilation caused by hyperglycemia in humans. Circ Res 2002, 90(1):107-11

42. Inoguchi T, Li P, Umeda F, Yu HY, Kakimoto M, Imamura M, et al: High glucose level and free fatty acid stimulate reactive oxygen species production through protein kinase C-dependent activation of $\mathrm{NAD}(\mathrm{P}) \mathrm{H}$ oxidase in cultured vascular cells. Diabetes 2000, 49(11):1939-45.

43. Pacher P, Beckman JS, Liaudet L: Nitric oxide and peroxynitrite in health and disease. Physiol Rev 2007, 87(1):315-424

44. Wolin MS, Gupte SA, Oeckler RA: Superoxide in the vascular system. J Vasc Res 2002, 39(3):191-207.

45. Balasubramanyam M, Adaikala Koteswari A, Sampath Kumar R, Finny Monickaraj S, Uma Maheswari J, Mohan V: Curcumin-induced inhibition of cellular reactive oxygen species generation: Novel therapeutic implications. J Biosciences 2003, 28(6):715-21.

\section{Pre-publication history}

The pre-publication history for this paper can be accessed here: http://www.biomedcentral.com/1472-6882/10/57/prepub

doi:10.1186/1472-6882-10-57

Cite this article as: Rungseesantivanon et al:: Curcumin supplementation could improve diabetes-induced endothelial dysfunction associated with decreased vascular superoxide production and PKC inhibition. BMC Complementary and Alternative Medicine 2010 10:57.

\section{Submit your next manuscript to BioMed Central and take full advantage of:}

- Convenient online submission

- Thorough peer review

- No space constraints or color figure charges

- Immediate publication on acceptance

- Inclusion in PubMed, CAS, Scopus and Google Scholar

- Research which is freely available for redistribution

Submit your manuscript at www.biomedcentral.com/submit
Ciomed Central 